\title{
Passive Earth Entry Vehicle Landing Test
}

\author{
Sotiris Kellas \\ NASA Langley Research Center \\ MS 489 \\ Hampton, VA 23681 \\ 757-864-4150 \\ Sotiris.Kellas@NASA.Gov
}

\begin{abstract}
Two full-scale passive Earth Entry Vehicles (EEV) with realistic structure, surrogate sample container, and surrogate Thermal Protection System (TPS) were built at NASA Langley Research Center (LaRC) and tested at the Utah Test and Training Range (UTTR). The main test objective was to demonstrate structural integrity and investigate possible impact response deviations of the realistic vehicle as compared to rigid penetrometer responses. With the exception of the surrogate TPS and minor structural differences in the back shell construction, the two test vehicles were identical in geometry and both utilized the Integrated Composite Stiffener Structure (ICoSS) structural concept in the forward shell. The ICoSS concept is a lightweight and highly adaptable composite concept developed at NASA LaRC specifically for entry vehicle TPS carrier structures.
\end{abstract}

The instrumented test vehicles were released from a helicopter approximately $400 \mathrm{~m}$ above ground. The drop height was selected such that at least $98 \%$ of the vehicles terminal velocity would be achieved. While drop tests of spherical penetrometers and a low fidelity aerodynamic EEV model were conducted at UTTR in 1998 and 2000, this was the first time a passive EEV with flight-like structure, surrogate TPS, and sample container was tested at UTTR for the purpose of complete structural system validation. Test results showed that at a landing vertical speed of approximately $30 \mathrm{~m} / \mathrm{s}$, the test vehicle maintained structural integrity and enough rigidity to penetrate the sandy clay surface thus attenuating the landing load, as measured at the vehicle CG, to less than $600 \mathrm{~g}$. This measured deceleration was found to be in family with rigid penetrometer test data from the 1998 and 2000 test campaigns.

Design implications of vehicle structure/soil interaction with respect to sample container and sample survivability are briefly discussed.

\section{TABLE OF Contents}

1. Introduction $\ldots \ldots \ldots \ldots \ldots \ldots \ldots \ldots \ldots \ldots \ldots \ldots$

2. Test Description ...................... 2

2.1 UTTR 1998 and 2000 Test Campaigns............. 2

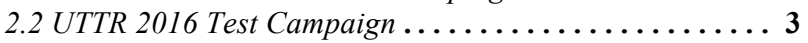

3. Discussion of Results .................. 5

3.1 UTTR 1998 and 2000 Test Results...............5

3.2 UTTR 2016 Test Results ......................6 6

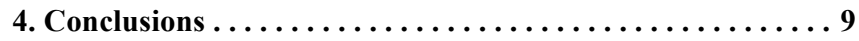

Acknowledgements ........................

References .......................... 10

Biography. ........................

U.S. Government work not protected by U.S. copyright

\section{INTRODUCTION}

In 1998 researchers at NASA LaRC introduced the passive (no parachute) Earth Entry Vehicle (EEV) for a Mars Sample Return (MSR) mission [1]. By eliminating all active systems, and relying solely on passive energy absorbers for landing-load attenuation, the passive EEV could meet much higher levels of reliability when compared with conventional entry vehicles [2]. For MSR, a very high level of reliability was driven by planetary sample containment for the purpose of planetary protection.

Due to changing NASA priorities the MSR mission was placed on hold in the early 2000. In the mid 2000's the passive EEV concept was resurrected with the development of a Multi-Mission Earth Entry Vehicle (MMEEV) concept [3]. The MMEEV work was then followed by the development of a Systems Analysis for Planetary Entry (SAPE) tool [4]. The present work represents a natural extension of the MSR EEV work with the goal to introduce structural fidelity to the MMEEV concept and investigate the effects of realistic structure on the impact response.

While advantages and disadvantages between parachuted versus chute-less EEVs have been reported in more detail elsewhere [5], from an impact point of view, the most important difference is the landing velocity. Lack of active systems to dissipate kinetic energy in the subsonic regime means that the passive EEV has to land at a much higher velocity compared to a parachuted vehicle. Higher landing velocity introduces extra structural and landing-energy absorbing design challenges for the vehicle.

In general, there are two distinct choices for landing energy dissipation [6]. The first is the one employed by the original MSR EEV [7 \& 8] and it is referred to as the "Racecar" energy absorbing method. The second is referred to as the "Camera-Case" method. These design approaches represent the two extremes of the trade space and practical designs in combination with actual landing sites may very well end-up somewhere in between the two.

The camera-case option is typically better suited to lower energy impacts but can be utilized in some high-energy applications, such as the passive EEV, provided that a significant portion of the kinetic energy can be absorbed 
through soil penetration. Assuming the forward shell maintains its integrity/shape during impact, the energyabsorbing event can be decoupled from the rest of the vehicle and hence the energy absorber can be sized and verified separately. Being able to size the energy absorber independently of the vehicle is the main advantage offered by the camera-case design approach. A major disadvantage/complexity associated with this approach is ensuring sample-container to energy-absorber attachment integrity throughout the impact event. Just like a car passenger with unfastened seatbelt during a crash, poor coupling and/or attachment failure between the sample container and the energy absorber during landing can result in much greater and unpredictable payload loading.

For sample return missions that utilize a passive EEV and do not have stringent sample containment requirements, a 3$\sigma$ landing ellipse could comfortably fit within UTTR's relatively uniform surface [5]. UTTR's sandy clay surface was characterized extensively in two separate test campaigns using rigid penetrometers in support of the original MSR project [1]. The first series of tests took place during the winter of 1998 and utilized a bucket-truck and a hot-air balloon to reach the desired drop heights. The second series took place in the summer of 2000 and utilized a helicopter to lift the test articles to the predetermined drop height. Results from these earlier studies highlighted the effectiveness of the sandy clay to dissipate impact energy. Furthermore, because of the presence of salt, UTTR's drylake surface remains relatively uniform throughout the year despite the wide variation in temperature and moisture. This unique property of the dry lakebed surface is evident in the 1998 photograph, Figure 1. While the access road was frozen the lakebed was not. Moreover, UTTR ground hardness was proven to be relatively unaffected by seasonal changes through the 1998 and 2000 test campaigns which took place during winter and summer, respectively.

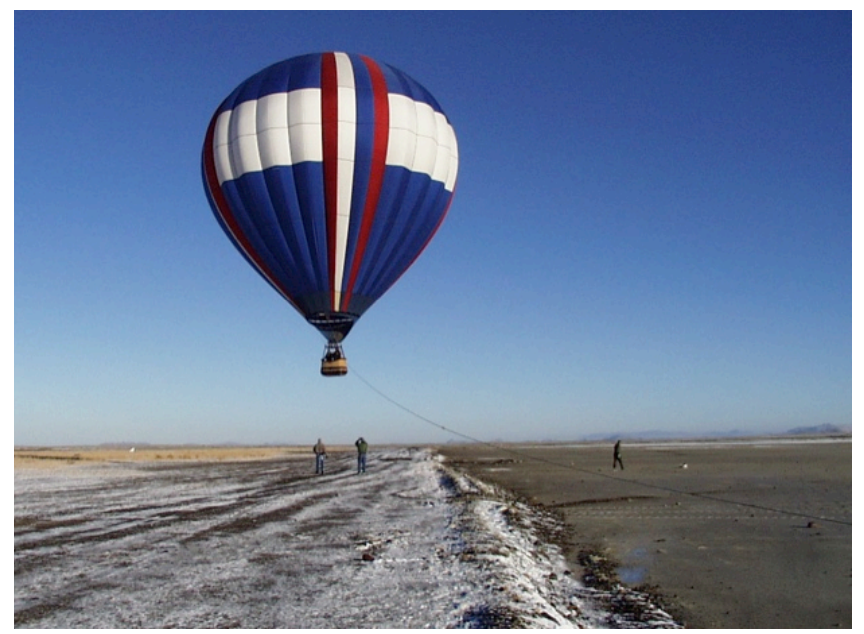

Figure 1. Hot-air balloon shown hovering over a UTTR access dirt-road. The lakebed is shown on the right

The relatively soft UTTR surface offers a unique opportunity to utilize a variation of the camera-case option and to rely solely on ground penetration for landing load attenuation. Taking advantage of ground penetration and eliminating the crushable energy absorber from the entry vehicle can lead to a number of design advantages and/or simplifications including (a) easier to achieve and maintain good payload to vehicle coupling which would be very hard to accomplish in a crushable design, (b) assuming that the forward shell remains sufficiently rigid during impact, easy to predict (based on rigid penetrometer data) peak g-loading, and (c) easier to predict the post-landing condition of samples due to thermal soak-back from the hot heatshield.

With the broad objective of demonstrating (a) good payload to vehicle coupling could be achieved with a lightweight design, and (b) peak g-loading could be predicated by rigid penetrometer data, two high fidelity EEVs measuring $1.2 \mathrm{~m}$ in diameter and utilizing a non-crushable payload support structure were designed and built at NASA LaRC and tested at UTTR.

With the exception of the surrogate Thermal Protection System (TPS) and minor structural differences in the aft shell construction, the two test vehicles were identical in geometry and both utilized the ICoSS structural concept in the fore shell [6]. ICoSS is a lightweight and highly adaptable composite concept developed at NASA LaRC for TPS carrier structures. The same manufacturing processes described in Reference [6] were also employed for all composite parts of the test vehicles.

A complete study including the ground characterization tests from the 1998 and 2000 test campaigns as well as the recent full-scale EEV tests is presented in this paper. Impact responses of the realistic test vehicle are compared against the instrumented rigid-penetrometer tests and implications of vehicle structure/soil interaction with respect to sample container and sample survivability are discussed.

\section{TEST DESCRIPTION}

\subsection{UTTR 1998 and 2000 Test Campaigns}

Several months prior to the 1998 UTTR ground characterization test campaign, the dry lakebed of UTTR was surveyed using handheld penetrometers. These consisted of $250 \mathrm{~mm}$ diameter hemispherical aluminum shells ballasted in the nose for stability and equipped with a self-contained data recorder with a triaxial accelerometer. The penetrometers were typically released from shoulder height to record the impact response. Spot checks at hundreds of different locations indicated that with the exception of a few manmade features, such as dirt access roads, the test range was otherwise flat and consisted of a relatively uniform-hardness soil. Therefore, more detail soil characterization at any given area was thought to be representative of the entire lakebed.

Because of the unique qualities of the dry lakebed, several attempts to formulate a surrogate clay material that could be used at LaRC to develop sufficient material models that 
could then feed dynamic finite element analysis simulations were unsuccessful. Consequently, representative scale test articles were designed and additional tests were planned and executed at UTTR.

The first detailed ground characterization series of tests was performed in the early winter of 1998 using a bucket truck and a hot-air balloon (Figure 1) to drop larger and heavier instrumented hemispherical penetrometers of different diameter and mass from predetermined heights to measure peak accelerations. The results revealed that the peak g-load (A) was proportional to the product of penetrometer Diameter (D) and impact velocity (V) squared over the penetrometer mass (M) as shown in Equation 1.

$$
A \propto D V^{2} / \mathrm{M}
$$

Therefore, based on an expected EEV mass, nose diameter, and impact velocity, peak vehicle impact acceleration, A, could be estimated. However, impact velocity limitations due to instrumentation full-scale capability, representative $D V^{2} / M$ values could not be achieved in 1998 series of tests. With improved instrumentation, higher impact velocity tests were planned and executed during the summer of 2000 to better match the MSR EEV parameters. A full-scale aerodynamic model test of the EEV with the objective to verify aerodynamic stability was also performed in 2000 . Because the hot-air balloon could not be used in high wind conditions and several days in 1998 were wasted waiting for favorable conditions, in 2000 a helicopter replaced the hotair balloon.

Three hemispherical rigid penetrometers were used to characterize the UTTR soil during the winter of 1998 and summer of 2000. All three penetrometers were made of common off-the-shelve spun aluminum shells, custom made reinforcing Bakelite ribs, Bismuth nose ballast, and a fourpoint lifting harness. One of the original $0.515 \mathrm{~m}$ diameter penetrometers is shown in Figure 2. Geometric details and mass settings for the three penetrometers are listed in Table 1.

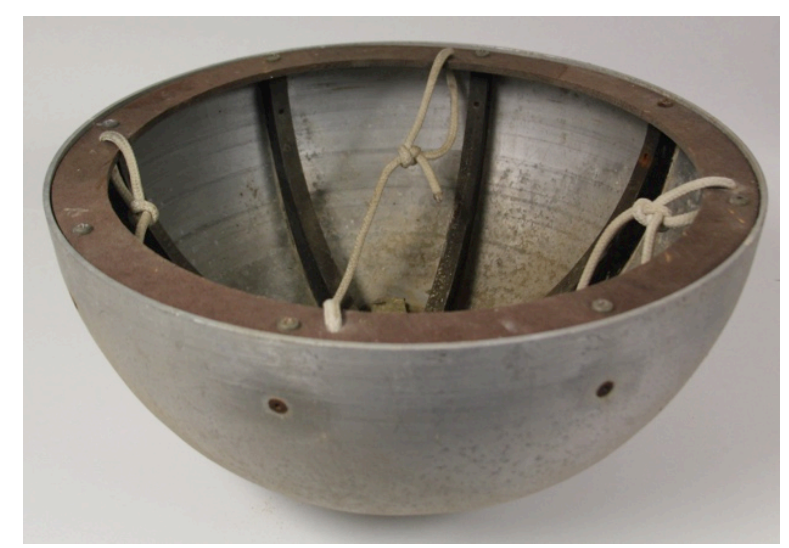

Figure 2. Photograph of an original penetrometer used for UTTR soil characterization during the 1998 and 2000 test campaigns
Table 1. Penetrometer geometry and mass

\begin{tabular}{|c|c|c|}
\hline $\begin{array}{c}\text { Diameter, } \\
\mathbf{m}\end{array}$ & $\begin{array}{c}\text { Shell Thickness, } \\
\mathbf{~ m m}\end{array}$ & $\begin{array}{c}\text { Mass Settings, } \\
\mathbf{k g}\end{array}$ \\
\hline \hline 0.660 & 3.2 & 15.29 or 24.08 \\
0.515 & 6.4 & 12.62 or 25.13 \\
0.402 & 3.2 & 12.05 or 24.50 \\
\hline
\end{tabular}

The cross section of the aerodynamic EEV model is shown in Figure 3. With the aid of a helicopter the instrumented test vehicle was released from a height of approximately $1200 \mathrm{~m}$ above the UTTR surface with the primary objective to demonstrate aerodynamic stability. The test vehicle's mass and moments of inertia were tailored to be as close to the MSR flight EEV as practically possible. However, the structure of the model was not at all representative of a flight configuration. With the exception of the instrumentation package that weighed about $1 \mathrm{~kg}$ and the ballast ring in the wing tip of the vehicle, about $90 \%$ of the vehicle mass was essentially devoted to ensuring structural integrity as opposed to less than $25 \%$ for a flight-like vehicle.

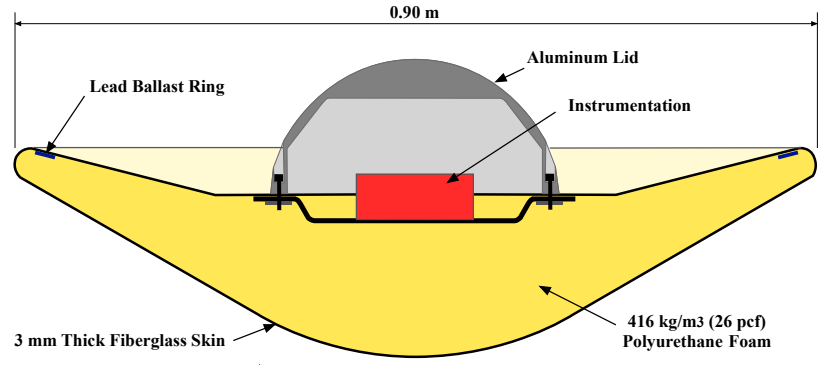

Figure 3. Cross-section schematic of the low-fidelity aerodynamic MSR EEV model

2.1.1 UTTR 1998 and 2000 Instrumentation-Penetrometer instrumentation for the 1998 campaign consisted of a selfcontained EDR-3 data recorder with a maximum data acquisition rate of $3.2 \mathrm{kHz}$ and triaxial accelerometer with a full-scale range of $500 \mathrm{~g}$. Because of the limits in the data rate and full-scale accelerometer range, 1998 test were restricted to relatively low impact speeds. To achieve higher impact speeds the instrumentation was upgraded in 2000. This consisted of a custom IES multi-trigger data recorder with $117.6 \mathrm{kHz}$ maximum data-rate capability and externally attached accelerometers - a 2-g full-scale to estimate the impact velocity and a 6000-g to measure the impact event. Multi-triggering allowed for free-fall data to be acquired at lower rates and avoid storage memory saturation. The unit switched to high speed data recording when triggered on impact.

A secondary objective of the aerodynamic full-scale test was to record the impact event. Therefore, the model was designed to accommodate the same instrumentation package that was used in the penetrometers. Though the impact event was recorded successfully, a big question remained on whether an EEV with realistic structure and specifically 
designed for impact would have responded differently. This question was finally answered with the test campaign of 2016 .

\subsection{UTTR 2016 Test Campaign}

2016 test objectives were (a) demonstrate that for a realistic $\mathrm{EEV}$, attachment integrity between the sample container and vehicle could be maintained during landing, (b) for a spherical and relatively rigid-nose EEV, peak g-loads could be predicated using rigid penetrometer data, and (c) provide test data for LS-Dyna landing simulation validation.

To achieve the above objectives, two high fidelity EEVs measuring $1.2 \mathrm{~m}$ in diameter and utilizing a non-crushable payload support structure were designed and built at NASA LaRC. The aerodynamic shape of the test articles was similar but slightly larger than the original MSR EEV. The manufacturing processes described in Reference [6] were also employed for all parts of the test vehicles. Essentially, composite materials pre-impregnated with high temperature matrices were substituted with lower cost epoxies.

A schematic of the 2016 test-vehicle's cross-section is shown in Figure 4. As well as realistic structure, both 2016 test vehicles included surrogate TPS, and a sample container with flight-like attachment to the payload support structure.

A structural difference, relatively insignificant with respect to impact, between the two vehicles was in the construction of their aft shell. The aft shell of test vehicle \#1 was constructed of 6-layers of graphite/epoxy polar-fabric versus 3-layers per skin and Rohacell-core sandwich for test vehicle \#2. This change was made purely for manufacturing demonstration purposes and depending on the choice of back-shell TPS could be more significant for a flight vehicle.

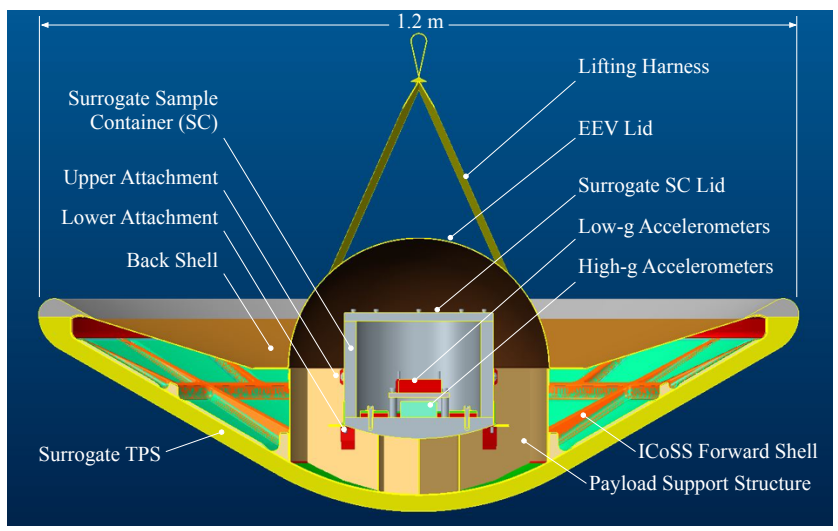

Figure 4. Test Vehicle \#1 cross-section schematic

Another difference between the two test vehicles was in the surrogate TPS. The chosen material for both vehicles was closed cell polyurethane foam of about the same density as PICA. Test vehicle \#1 was fitted with a relatively tough version to minimize crushing and maintain the spherical nose shape during impact and to provide data for direct comparison with rigid penetrometer data. The second vehicle was fitted with frangible foam to investigate the effect of TPS crushing on the landing response and to provide an additional degree of fidelity for future LS-Dyna model validation. Nominal densities for the two materials were 320 and $288 \mathrm{~kg} / \mathrm{m}^{3}$ for test vehicles \#1 and \#2, respectively.

To ensure that landing energy was dissipated primarily by soil penetration both the ICoSS forward shell and the payload support structure were designed for toughness and rigidity. In a typical building block fashion, and prior to any payload-support-structure/EEV integration, several forward ICoSS shells and payload support structures were manufactured and tested individually using static loading. Development of the forward shell was reported in Reference [6].

In addition to static testing, a prototype payload support structure was also loaded dynamically up to $1500 \mathrm{~g}$ to confirm a structure weighing less than $0.8 \mathrm{~kg}$ could maintain structural integrity while reacting the dynamic loads generated by the sudden deceleration of up to $14.5 \mathrm{~kg}$ of payload travelling in excess of $30 \mathrm{~m} / \mathrm{s}$. The $1500 \mathrm{~g}$ limit was chosen based on past MSR sample integrity studies which indicated that rock samples could retain their integrity and hence science value when subjected up to these loads for a short period of time.

Maximum specific stiffness and stability in the payload support structure were achieved by the use of graphite fiber reinforced epoxy. Derived from earlier efficient concepts [7], the payload support structure was designed with a series of intersecting graphite/epoxy webs. An isometric view of the structure is shown in Figure 5. As well as being lightweight the chosen design is also highly tailorable capable of meeting evolving mission requirements. Design features that can be easily modified include (a) with appropriate use of fiber-reinforcement hybridization the rigid support structure can be transformed into a crushable structure, and (b) can be sized to react any load level by modifying the thickness and/or total number of intersecting webs.

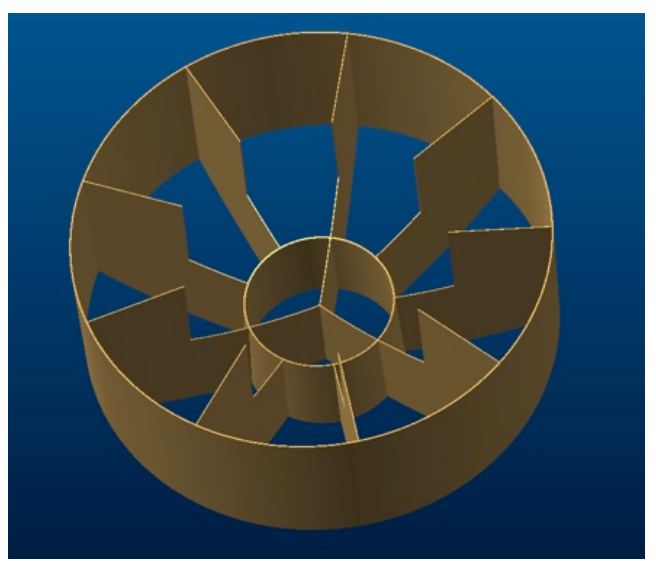

Figure 5. CAD of Payload Support Structure (PSS) 
2.2.1 UTTR 2016 Instrumentation-Two sets of triaxial accelerometers were located within the sample container as shown in Figure 4. A 6000-g 6DXP data recorder was mounted directly on the base of the container to capture the impact event at $75 \mathrm{kHz}$, and a 20 -g TSR Pro data recorder was positioned close to the vehicle's center of gravity.

To capture the vehicle attitude at impact a set of high-speed Phantom 10 cameras were placed approximately $200 \mathrm{~m}$ from the center of a predetermined impact zone at right angles to each other. The impact zone diameter was defined by the camera field of view and was approximately equal to $60 \mathrm{~m}$. An onboard camera was mounted to the belly of the helicopter and was pointed down to capture the hook-release event.

To aid photogrammetric analysis the test vehicles were painted black and white using a pattern that was unique when viewed along a given orientation.

Each test vehicle was released from the helicopter over the marked impact zone. The drop height of approximately 400 $m$ was selected such that it was high enough to ensure at least $98 \%$ of the vehicles terminal velocity would be reached while also being low enough to maximize the chance that the test vehicles would land within the predefined impact zone. The GPS position of the center of the impact zone and ground wind direction and speed were relayed to the helicopter pilot.

To verify that landing in the $60 \mathrm{~m}$ diameter impact zone was possible, a series of preliminary drop tests were conducted utilizing one of the aluminum penetrometers used in the summer of 2000 test campaign. Unfortunately, and despite successful practice runs, the combination of progressively worsening wind conditions and difficulty positioning the helicopter correctly prior to release resulted in only one of the test vehicle drops being successful.

The EEV test article is shown resting on the ground and connected to the helicopter by $30-\mathrm{m}$ long umbilical cord prior to liftoff in Figure 6.

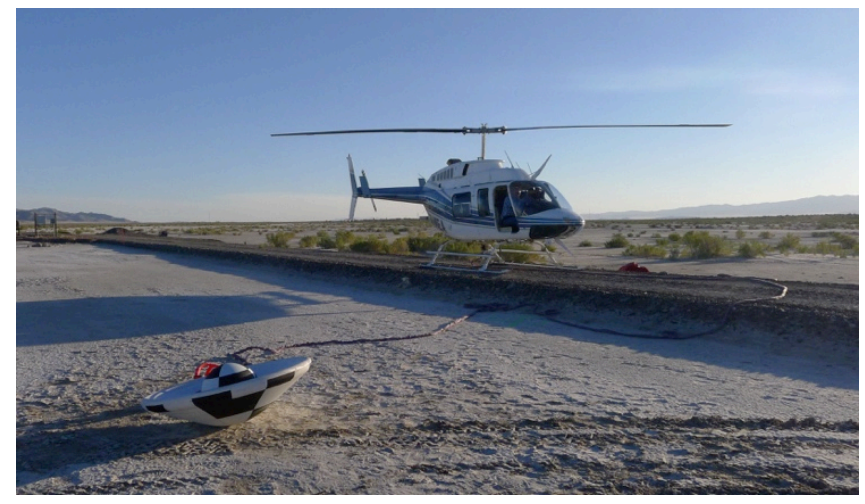

Figure 6. Test Vehicle\#2 on the ground prior to liftoff

\section{DISCUSSION OF RESULTS}

\subsection{UTTR 1998 and 2000 Test Results}

A typical penetrometer response is shown in Figure 7 and a summary of the rigid penetrometer tests from the MSR UTTR ground characterization studies is presented in Table 2. Peak dynamic load in Table 2 was determined from the filtered impact response at $1 \mathrm{kHz}$.

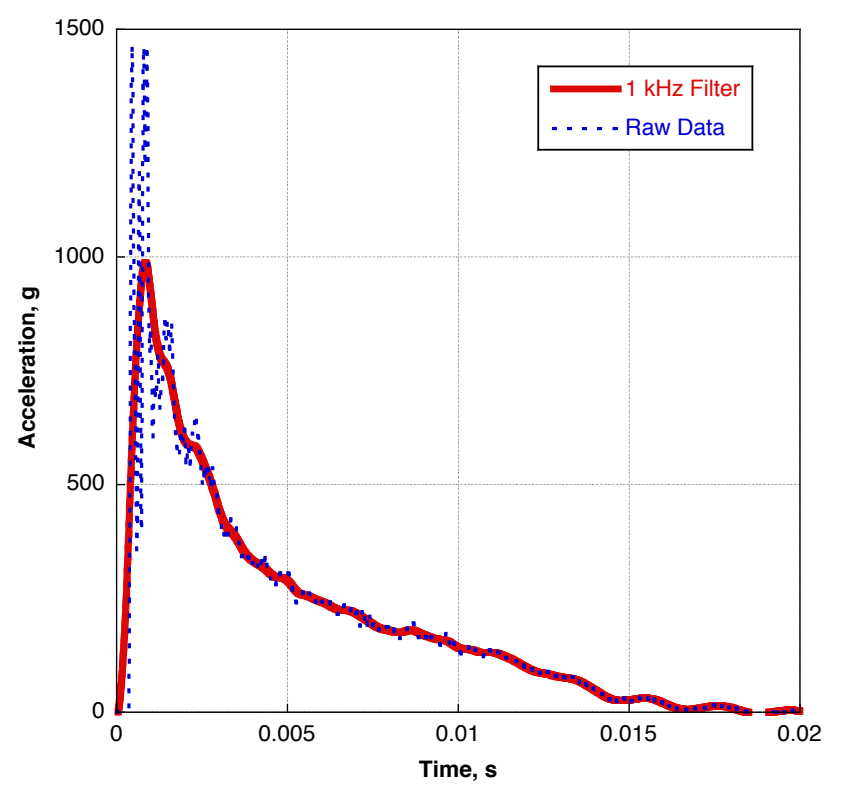

Figure 7. Typical impact response a hemispherical and rigid penetrometer with diameter, mass, and impact velocity equal to $0.514 \mathrm{~m}, 25.13 \mathrm{~kg}$, and $39.7 \mathrm{~m} / \mathrm{s}$, respectively

The assumption of penetrometer rigidity appeared to hold relatively well and typically natural frequencies were high enough that the application of a $1 \mathrm{khz}$ low pass filter was sufficient to clean the dynamic response as shown in Figure 7. A simple data filtering metric that was employed in all acceleration/time responses, involved integration of the raw and filtered acceleration data to estimate the area under the acceleration/time curve. The filtered signal at a given low pass frequency was deemed acceptable if all high frequency vibration was removed and the difference in area (velocity) between the raw and filtered accelerations was less than $1 \%$.

The full-scale aerodynamic model however was not designed for impact and isolating the structural response from vibration noise was not as straightforward as in the case of the rigid penetrometers. Furthermore, an inadvertent release of the model, caused by a violent swing of both hook and model, sent the test article tumbling to the ground from an altitude of nearly $1000 \mathrm{~m}$. The model landed upside down and appeared externally to have survived the impact. However, separation between the skin and the foam core, particularly at the instrumentation attachment area, may have occurred. Because the vehicle was deemed 
aerodynamically sound, a second drop was attempted. In the second test attempt the helicopter ascended at a much lower climb rate to avoid the earlier issue. Following a clean release, the model exhibited the same aerodynamic stability that scaled models displayed in the NASA Langley spin tunnel months earlier [9].

Table 2 Penetrometer Test Results

\begin{tabular}{|c|c|c|c|c|}
\hline $\begin{array}{c}\text { Test } \\
\text { Method }\end{array}$ & $\begin{array}{c}\text { Diam., } \\
\mathbf{m}\end{array}$ & $\begin{array}{c}\text { Vel. } \\
\mathbf{m} / \mathbf{s}\end{array}$ & $\begin{array}{c}\text { Mass, } \\
\text { kg }\end{array}$ & $\begin{array}{c}\text { Peak } \\
\text { Load, }\end{array}$ \\
\hline \hline B. Truck & 0.514 & 11.02 & 22.2 & 790 \\
B. Truck & 0.514 & 18.54 & 19.8 & 309 \\
B. Truck & 0.514 & 11.02 & 18.6 & 435 \\
B. Truck & 0.2 & 2.98 & 19.5 & 370 \\
B. Truck & 0.514 & 18.54 & 17.2 & 216 \\
B. Truck & 0.514 & 18.54 & 17.5 & 236 \\
Balloon & 0.514 & 18.91 & 22.9 & 320 \\
Balloon & 0.514 & 18.91 & 23.2 & 405 \\
Balloon & 0.514 & 18.91 & 27.7 & 450 \\
Balloon & 0.514 & 18.91 & 27.3 & 510 \\
Helicopter & 0.400 & 12.05 & 35.0 & 1194 \\
Helicopter & 0.400 & 12.05 & 43.2 & 1483 \\
Helicopter & 0.400 & 12.05 & 44.9 & 1659 \\
Helicopter & 0.400 & 24.50 & 31.9 & 613 \\
Helicopter & 0.400 & 24.50 & 39.6 & 813 \\
Helicopter & 0.400 & 24.50 & 45.4 & 1017 \\
Helicopter & 0.514 & 25.13 & 42.2 & 1164 \\
Helicopter & 0.514 & 25.13 & 39.7 & 988 \\
Helicopter & 0.514 & 25.13 & 42.4 & 977 \\
Helicopter & 0.514 & 25.13 & 40.4 & 916 \\
Helicopter & 0.514 & 25.13 & 41.2 & 1037 \\
Helicopter & 0.514 & 25.13 & 47.9 & 1326 \\
Helicopter & 0.660 & 24.08 & 35.1 & 1080 \\
Helicopter & 0.660 & 24.08 & 40.7 & 790 \\
\hline
\end{tabular}

The aerodynamic test vehicle had a nose diameter of 0.704 $\mathrm{m}$, a mass of $39.36 \mathrm{~kg}$ and impacted at an estimated velocity of $39.7 \mathrm{~m} / \mathrm{s}$. Given these parameters, $D V^{2} / M$ from Equation 1 was equal to $28.19 \mathrm{~m}^{3} / \mathrm{kg}^{*} \mathrm{~s}^{2}$. The impact response including filtered data at two low pass filter frequencies is shown in Figure 8.

Due to lack of structural rigidity as well as suspected delamination from the inadvertent first drop, the typical $1 \mathrm{kHz}$ filter frequency was not sufficient to remove all high frequency vibration from the acceleration response. As shown in Figure 8, a more suitable low pass filter frequency was $0.5 \mathrm{kHz}$. The resulting peak acceleration based on the $0.5 \mathrm{kHz}$ filtered data was $1100 \mathrm{~g}$.

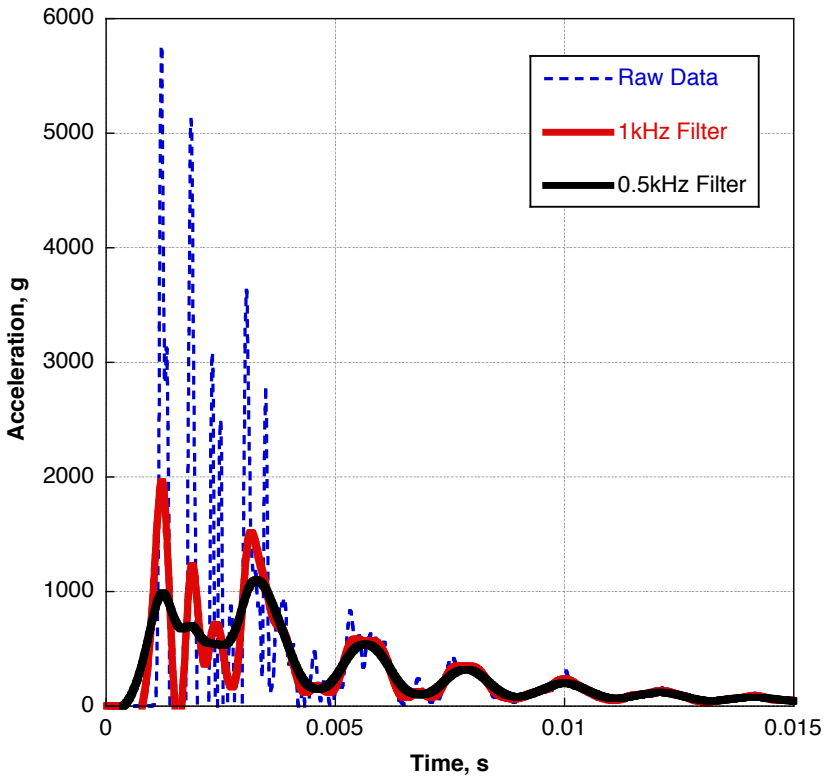

Figure 8. MSR aerodynamic test vehicle Impact response

\subsection{UTTR 2016 Test Results}

Despite preliminary practice drop tests, landing the test vehicles within the predefined $60 \mathrm{~m}$ diameter landing-zone proved challenging. The combination of gusty wind conditions, inaccurate helicopter position at release and erratic hook release resulted in only one successful impact test - the first test of test vehicle \#1.

With the sample container ballasted at $12.5 \mathrm{~kg}$, test vehicle \#1 was released from an altitude of $400 \mathrm{~m}$ above the UTTR surface. At the time, a SSE wind, measured on the ground, was gusting to 10 knots. The test vehicle was released approximately $65 \mathrm{~m} \mathrm{NE}$ of the center of the impact zone and landed approximately $46 \mathrm{~m} \mathrm{NNW}$ of the release point and in the field-of-view of only one of the two high-speed cameras. However, the distance of the impact point from the camera of nearly $300 \mathrm{~m}$ was longer than ideal. When released from the helicopter-hook the vehicle was almost instantaneously perturbed by nearly $90^{\circ}$ off-axis as captured by the onboard cameras and shown in Figure 9. The vehicle eventually settled at an oscillation frequency of about $0.8 \mathrm{~Hz}$ as it approached the ground and landed with an attitude of approximately $4.8^{\circ}$ to the vertical.

The vertical impact velocity was measured using photogrammetry as well as by integrating the vertical acceleration. Using the integrated impact accelerations and correcting for the small off-axis orientation, the vertical impact velocity component was estimated at $29.6 \mathrm{~m} / \mathrm{s}$ and the horizontal component at $5.1 \mathrm{~m} / \mathrm{s}$. The total vehicle mass was $34.3 \mathrm{~kg}$, resulting in a $D V^{2} / M$ equal to $23.25 \mathrm{~m}^{3} / \mathrm{kg}^{*} \mathrm{~s}^{2}$. 

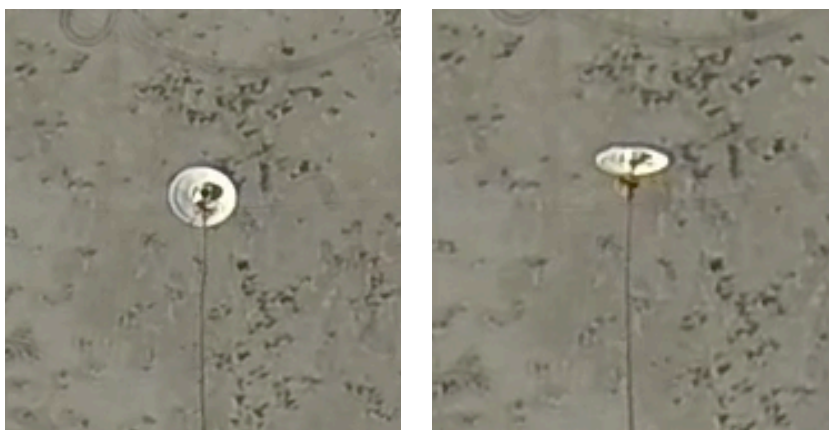

Figure 9. Video frames before (left) and after (right) test vehicle release from the helicopter.

Study of all videos revealed that similar issues at release also occurred in the subsequent two drop tests. Because the helicopter always traveled against the wind to the predefined release point and all test vehicles at release rotated about a horizontal axis normal to the helicopter's travel path, it is very likely that a head wind induced lift force caused the sudden vehicle rotation. When the vehicle was attached to the helicopter hook the lift force acting in the front half of the vehicle was reacted by the three-point harness and became unbalanced as soon as the vehicle was released.

The oscillation decay of the vehicle was confirmed by the on board low-g accelerometer responses as shown in Figure 10. For clarity, the $\mathrm{Y}$ acceleration component is not shown in Figure 10. This response was same in magnitude but out of phase to the acceleration component $\mathrm{X}$.

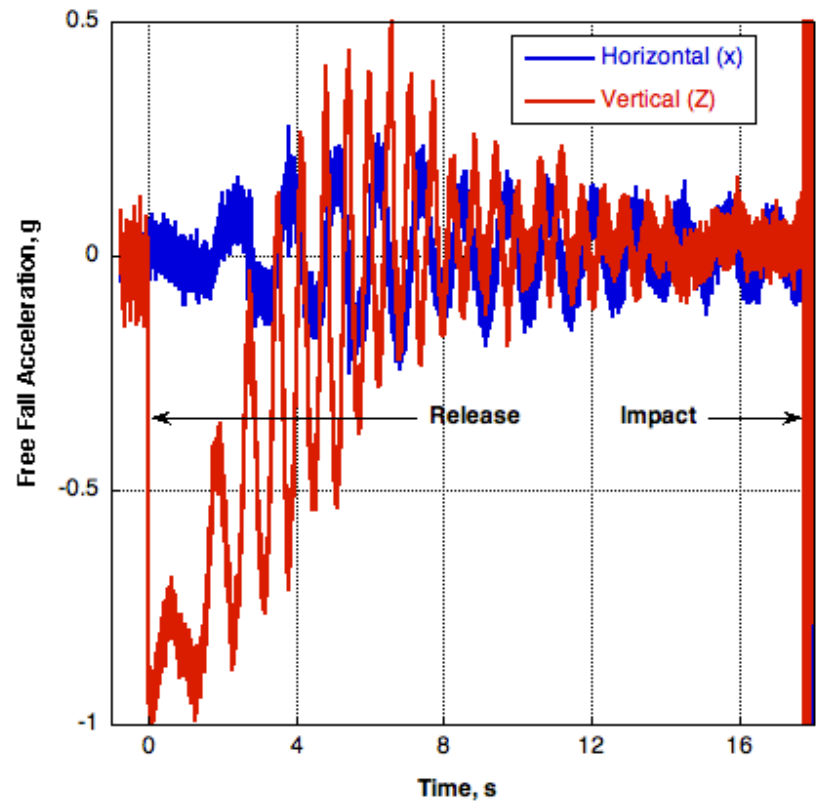

Figure 10. Test 1 free fall response as measured by on board low-g accelerometers.

The free fall responses of Figure 10 indicate that following release and initial perturbation, vehicle oscillations began to decay and settled into a stable pattern approximately 4 seconds prior to impact. Oscillation decay is an indication of vehicle dynamic stability [9].

A photograph of the test vehicle after landing is shown in Figure 11. Following landing the test vehicle was carefully inspected for structural damage. With the exception of minor epoxy-fillet cracks at three locations where the payload-support-structure webs make contact with the spherical nose of the vehicle, the rest of the structure appeared intact. In particular, all critical attachment points between the sample container and the payload support structure maintained complete integrity. Furthermore, no permanent deformation was evident in the surrogate TPS. The impact response of test 1 is presented in Figure 12 which shows that like the rigid penetrometers, random vibrations were attenuated with a low pass $1 \mathrm{kHz}$ filter. The measured peak load from filtered data at $1 \mathrm{kHz}$ or $0.5 \mathrm{kHz}$ was found to be $560 \mathrm{~g}$.

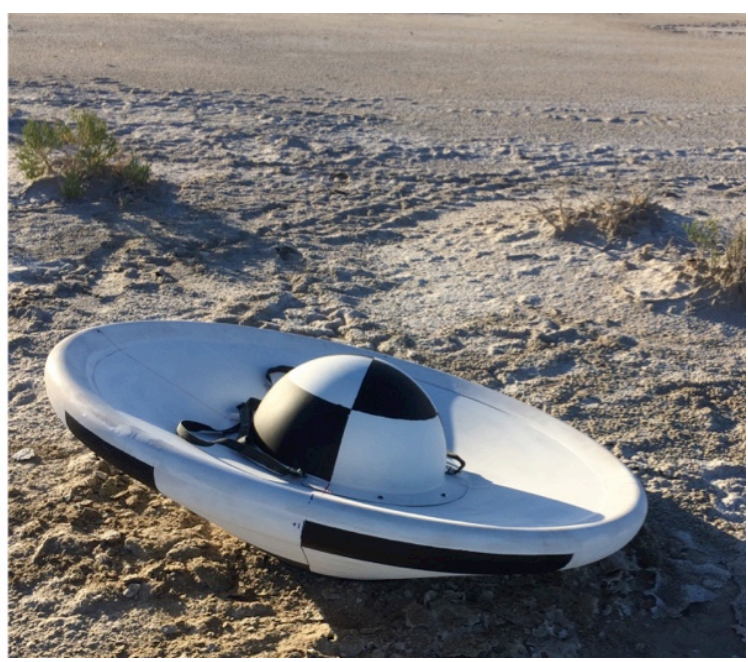

Figure 11. Photograph of test vehicle 1 following a 29.6 $\mathrm{m} / \mathrm{s}$ impact on UTTR soil.

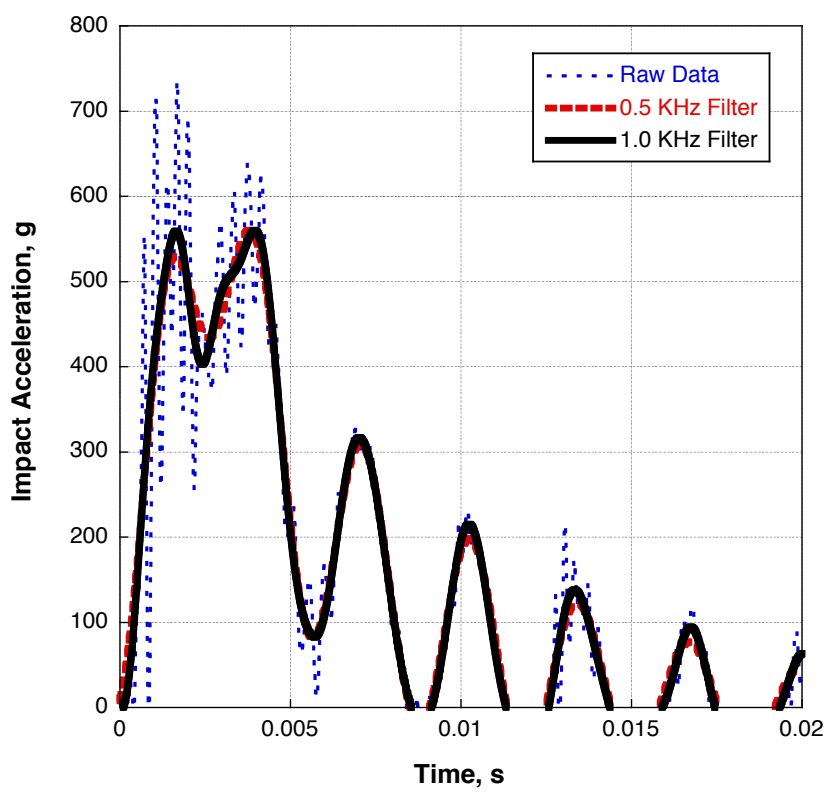

Figure 12. Test vehicle 1 - test 1 , impact response 
After inspection, test vehicle \#1 was prepared for a second test with the sample container ballast remaining at $12.5 \mathrm{~kg}$. Released with worse initial perturbation than the first test, the vehicle immediately flipped over and began tumbling. It eventually impacted the ground upside down, bounced, and came to rest adjacent to the primary impact location as shown in Figure 13.

With the exception of crushing of the hemispherical lid, which was not intended to resist landing loads, the rest of the structure maintained integrity. While tumbling of the test vehicle resulted in a no-test, close examination of the structure and the sample-container to payload-supportstructure attachment, which by now have experienced two impacts, further confirmed the robustness of the structural design.

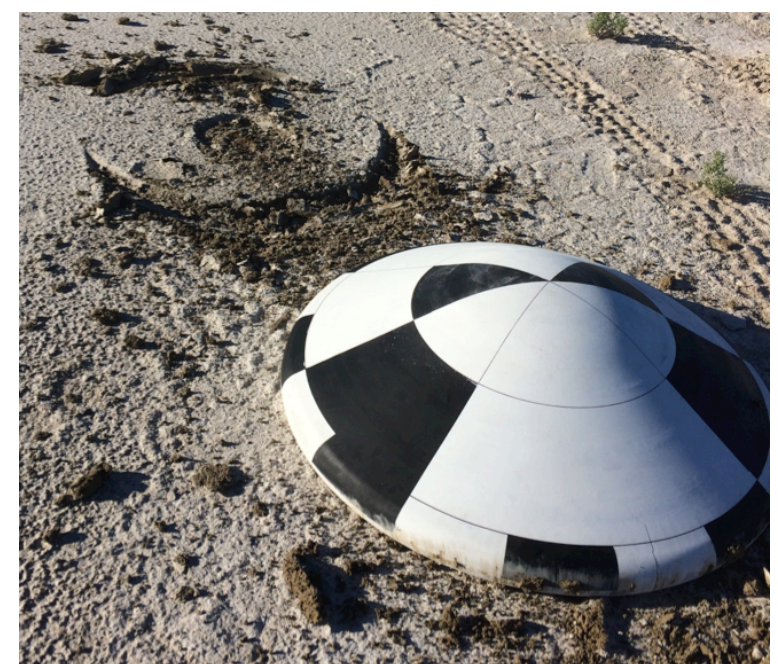

Figure 13. Photograph of test Vehicle 1 following a second $400 \mathrm{~m}$ drop on UTTR surface. The impact imprint is shown at the top left corner.

It is worth noting that a no-test was declared because tumbling of the vehicle is uncharacteristic of a flight EEV for (a) there is no mechanism by which, in nominal flight, the vehicle can experience a perturbation similar to the test, (b) both subscale [9] and full-scale vehicle aerodynamic tests have demonstrated dynamic stability during nominal conditions and (c) the flight EEV will also be spin stabilized at release from the spacecraft to improve vehicle stability even further.

The second test-vehicle was also ballasted with the sample container weighing $12.5 \mathrm{~kg}$ and dropped only once. This test too was declared a no-test. Again, test vehicle perturbation at release induced a high amplitude oscillation. While the vehicle did not tumble, the free-fall duration (due to the chosen low altitude) was too short to allow sufficient time for the oscillation to damp out, see Figure 14.

Because limit oscillation condition was not achieved by the time of impact, the vehicle landed on its shoulder with a relatively high horizontal and rotational velocity which caused the test article to bounce out of the impact crater and come to rest nose down, as shown in Figure 15.

While the landing was not representative of a flight vehicle and the test was declared a no-test, it did provide additional confirmation of the structural design robustness and the effectiveness of the sample-container to payload-supportstructure attachment to hold the $12.5 \mathrm{~kg}$ canister rigidly in place at any impact orientation.

At the completion of the full-scale tests, additional soil tests were conducted using a hand held hemispherical penetrometer to characterize the soil around the impact crater. These in situ test data will be used to tune the soil model even further in future LS-Dyna test simulations.

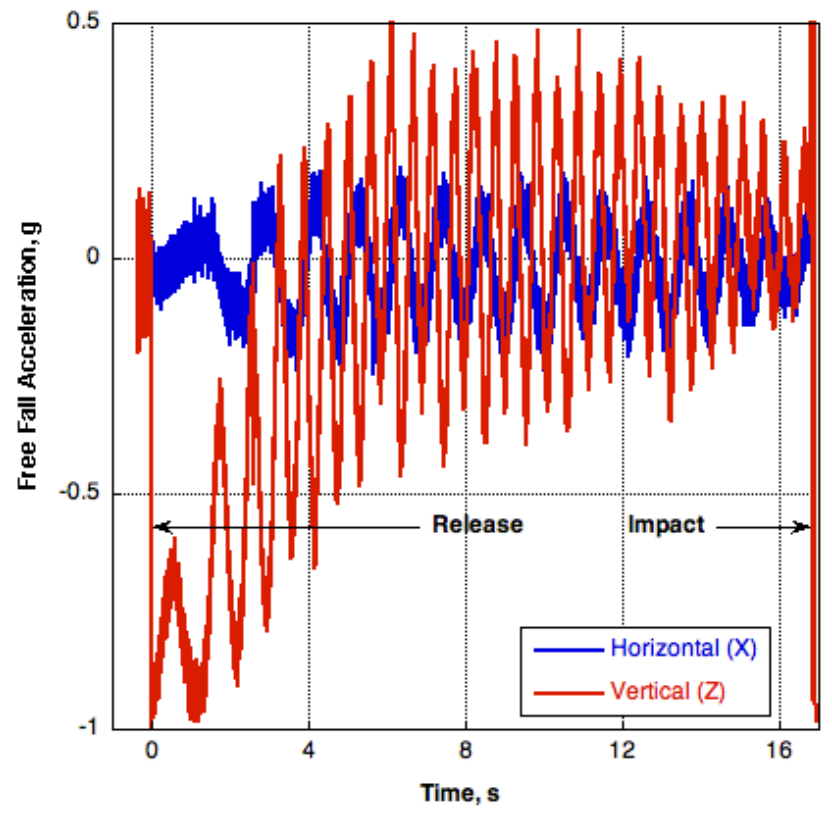

Figure 14. Test 3 free fall response as measured by on board low-g accelerometers.

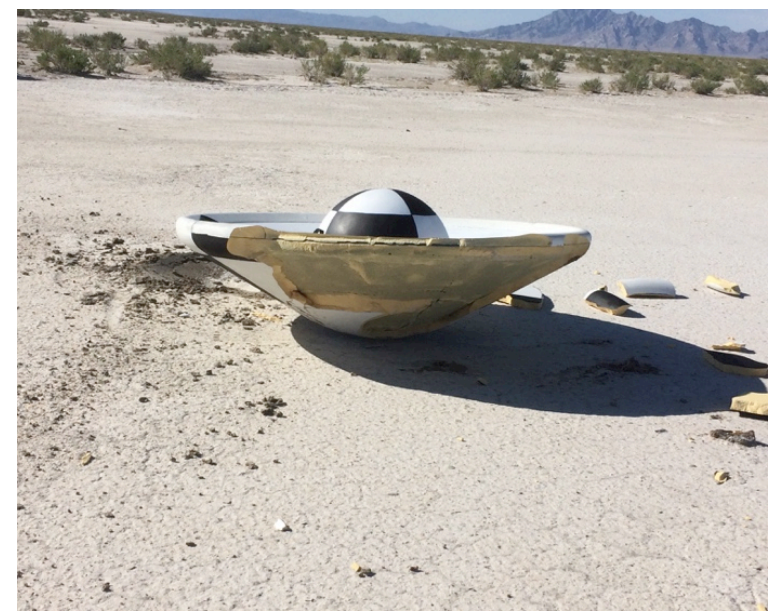

Figure 15. Photograph of test vehicle 2 following a $400 \mathrm{~m}$ high drop on UTTR surface. The camera was pointed at the area on the shoulder of initial impact 
One of the questions that the 2016 test campaign set out to answer was whether data from penetrometer tests could be relied on to predict the peak dynamic loads in a realistic EEV. To demonstrate that rigid penetrometer responses follow the relationship of Equation 1, data from Table 2 were used to calculate corresponding $D V^{2} / M$ values and plotted against peak load. The result is presented in Figure 16. Because the structural and aerodynamic EEVs had relatively low $D V^{2} / M$ values $\left(23.25\right.$ and $28.19 \mathrm{~m}^{3} / \mathrm{kg}^{*} \mathrm{~s}^{2}$ respectively), penetrometer data with $D V^{2} / M$ values greater than $50 \mathrm{~m}^{3} / \mathrm{kg}^{*} \mathrm{~s}^{2}$ (those which correspond to the two low mass / high speed tests of the $0.4 \mathrm{~m}$ diameter penetrometer) are not plotted in Figure 16.

The slope of the best linear fit of the penetrometer data was found to be $30 \mathrm{~g}^{*} \mathrm{~s}^{2} \mathrm{~kg} / \mathrm{m}^{3}$. Superimposed in the plot of Figure 16 are two points corresponding to the structural and aerodynamic EEVs. While both EEV points are in family with the rigid penetrometer data, the structural model appears to correlate better to penetrometer data due to its superior structural integrity. With the constant of proportionality equal to $30 \mathrm{~g} * \mathrm{~s}^{2} * \mathrm{~kg} / \mathrm{m}^{3}$, Equation 1 can be rewritten as:

$$
A=30 D V^{2} / \mathrm{M}
$$

The results of this study have clearly demonstrated that the empirical relationship, Equation 2, can be utilized to estimate the peak landing loads for any EEV (with $D V^{2} / M$ value up to $50 \mathrm{~m}^{3} / \mathrm{kg}^{*} \mathrm{~s}^{2}$ ) landing within UTTR's dry lakebed.

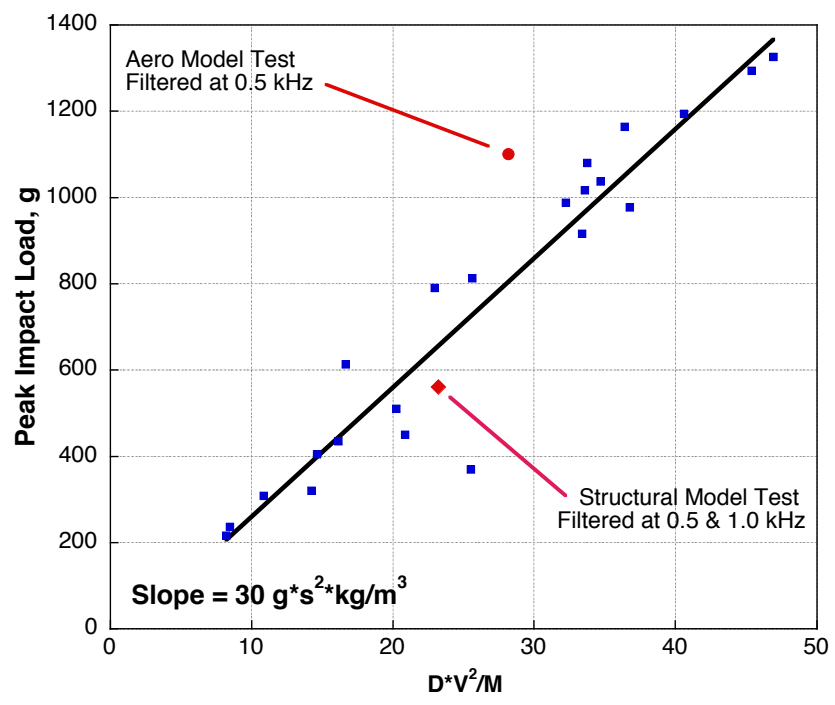

Figure 16. Peak dynamic load versus $D V^{2} / M$ for rigid penetrometer tests at UTTR

One however must not forget that use of Equation 2 is constrained to:

a) UTTR landings.

b) EEV nose is spherical, rigid, and the effect of TPS crushing is negligible in altering that nose shape. c) The vehicle behaves like a single mass throughout the impact event, and

d) The magnitude of the proportionality constant depends on the units used.

Any deviations from the above assumptions $\mathrm{b}$ and $\mathrm{c}$ will have to be analyzed using validated dynamic FEA models. More importantly, one must always remember that Equation 2 is predicting vehicle peak deceleration. Depending on how well any samples are packaged/restrained within the sample container, sample loading may or may not be the same as the vehicle load.

With a NASA Mars Sample Return demonstration mission scheduled to possibly occur within the next decade, a relevant question is how suitable this work is to such a mission? Clearly, the answer is highly dependent on mission reliability requirements.

With the mission failure (loss of sample containment) goal for the original MSR mission of less than 1 in a million, and the likelihood of man-made objects within the UTTR landing ellipse, the need to design against hard surface impacts preclude the design approach described in this paper and the "racecar" [6] approach will be the more appropriate solution. Nonetheless, primary structural elements such as the ICoSS forward shell structure and the payload support structure can easily be adapted to the racecar approach and serve the new MSR mission well.

For missions with more conventional reliability requirements (e.g. acceptable failure rates of less than 1 in a thousand) are likely to benefit greatly by taking advantage of the UTTR soft soil and utilizing the validated design presented here.

\section{Conclusions}

The work presented in this paper confirmed that for an EEV with spherical nose and landing within UTTR's dry lakebed, the vehicle peak landing load can be estimated using the empirical relationship of Equation 2.

Despite the fact that two out of three drop tests were classified no-test, the single successful test demonstrated the effectiveness of the ICoSS concept in meeting the nose rigidity assumption. The tests also verified the robustness of the payload support structure and its attachments to the sample-canister. Most importantly, the payload support system also proved to be robust for off-nominal impacts upside down impact in test 2 and shoulder impact in test 3 .

\section{ACKNOWLEDGEMENTS}

The author gratefully acknowledges the support provided by the following individuals: Mr. Donald Smith of NASA Langley for leading the test vehicle fabrication, Mr. Nicholas Vitullo of AMA for leading the Pro-E modeling, Dr. Justin Littell of NASA Langley for leading the test vehicle instrumentation and supporting the tests, and $\mathrm{Mr}$. 
David Paddock also of NASA Langley for providing Fibersim support.

\section{REFERENCES}

[1] Mitcheltree R. A., Kellas S., Dorsey J. T. Desai P. N, and Martin C. J., "A Passive Earth-Entry Capsule for Mars Sample Return,” AIAA Paper 98-2851

[2] Atkins K. L., Brownlee D. E., Duxbury T., Yen C. and Tsou P., "STARDUST: Discovery's Interstellar Dust and Cometary Sample Return Mission," Proceedings 1997 IEEE Aerospace Conference, Feb. 1997

[3] Maddock, R. W., "Sample Return Challenges MultiMission Earth Entry Vehicle Design Trade Space and Concept Development Strategy," 6th International Planetary Probe Workshop, Atlanta, GA, June 2008.

[4] Samareh, J. A., "A Multidisciplinary Tool for Systems Analysis of Planetary Entry, Descent, and Landing (SAPE)," NASA-TM-2009-215950

[5] Maddock R. W., Henning A., and Samareh J. A., "Passive vs. Parachute System Architecture for Robotic Sample Return Vehicles," paper 2087, IEEE Aerospace Conference, 2016

[6] Kellas S., "Integrated Composite Stiffener Structure (ICoSS) Concept for Planetary Entry Vehicles," 57th AIAA/ASCE/AHS/ASC Structures, Structural Dynamics, and Materials Conference, San Diego California, Jan. 2016.

[7] Kellas S., "Design, Fabrication and Testing of a Crushable Energy Absorber for a Passive Earth Entry Vehicle," NASA/CR-2002-211425

[8] Kellas S. and Mitcheltree R. A., "Energy Absorber Design, Fabrication and Testing for a Passive Earth Entrée Vehicle," AIAA-2002-1224

[9] Mitcheltree R. A, Fremaux C. M., and Yates L. A., "Subsonic Static and Dynamic Aerodynamics of Blunt Entry Vehicles," AIAA 99-1020

\section{BIOGRAPHY}

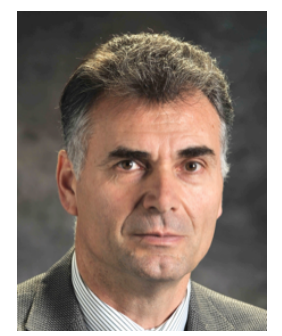

Sotiris Kellas received a B.S., in Aeronautical Engineering, an M.S. in Structural Mechanics and a Ph.D. in Composite Material and Structures from Imperial College, London England. He has been with NASA Langley for nine years. He began his career in 1988 as Visiting Assistant Professor at Virginia Tech before joining Lockheed Martin and then General Dynamics to provide research support for NASA.
He has served as the impact dynamics lead for the MSR $E E V$, and has extensive experience in the design, fabrication and testing of composite crushable energy absorbers. He was the test lead for many high profile NASA projects such as the Composite Crew Module, Composites for Explorations, and the Alternate Heatshield Carrier Structure of the Orion crew module. In addition to other inventions, he is the sole inventor of the Rigid Deployable Honeycomb and has published dozens of technical papers and NASA reports in the area of composite structures design, fabrication and testing. Currently, he is leading the development of the ICoSS structural concept for planetary entry vehicles. 
